\title{
Incomplete preference matrix with elements from an Alo-group and its application to ranking of alternatives
}

\author{
Jaroslav Ramík \\ Silesian University, School of Business Administration, Karvina CZ 73340, Czech Republic
}

\begin{abstract}
A preference matrix is the result of pairwise comparison and is a powerful method in multi-criteria optimization. When comparing two elements, the decision maker assigns the value from a given scale which is a linearly ordered Abelian group (Alogroup) to any pair of alternatives representing the element of the preference matrix (P-matrix). The well known multiplicative, additive and fuzzy preference matrices are generalized. Some situations where elements of the P-matrix are missing are focused on and a general method for completing a Pmatrix with missing elements called the extension of the P-matrix is proposed. Two important particular cases of fuzzy P-matrix with missing elements are discussed. Some illustrative numerical examples are given.
\end{abstract}

Keywords: Multi-Criteria Optimization, Pairwise Comparison, Preference Matrix, Incomplete Matrix, Alo-group.

\section{Introduction}

In various selection and prioritization processes the decision maker(s) (DM) try to find the best alternative(s) from a finite set of alternatives. DM problems and procedures have been established to combine opinions about alternatives related to different DM criteria. These procedures are often based on pairwise comparisons, in the sense that the processes are linked to some preference values from a given scale of one alternative over another. According to the nature of the information expressed by the DM, for every pair of alternatives different representation formats can be used to express preferences, e.g. multiplicative preference relations, [14], fuzzy preference relations, see [10], [13], [18], interval-valued preference relations, [34], and also linguistic preference relations, see [1].

In this paper pairwise comparison matrices over an Abelian linearly ordered group (Alo-group) are considered and, in this way, a general framework for all the above mentioned cases is provided. By introducing this more general setting, a consistency measure that has a natural meaning is proposed. It corresponds to the consistency indices presented in the literature, see e.g. [24] and is easy to calculate it in the additive, multiplicative and fuzzy cases. This setting is based on the works of [6], [7], and [24].

Usually, experts are characterized by their own personal background and experience of the problem to be solved. Expert opinions may differ substantially, some of them would not be able to efficiently express a preference degree between two or more of the available options. This may be true due to an expert not possessing a precise or sufficient level of knowledge of part of the problem, or because these experts are unable to discriminate the degree to which some options are better than others. In these situations such an expert will provide an incomplete preference matrix, see [1], [17], [34].

Usual procedures for DM problems correct this lack of knowledge of a particular expert using the information provided by the rest of the experts together with aggregation procedures, see [26]. In the literature, see [35], the problem is solved by the least deviation method to obtain a priority vector of corresponding the preference relation. In this paper, a general procedure that attempts to estimate the missing information in any of the above formats of incomplete preference relations is put forward. This proposal is different from the above mentioned procedures in [1], [17], [34] because the estimation of missing values in an expert incomplete preference matrix is done using only the preference values provided by these particular experts. By doing this, we assume that the reconstruction of the incomplete preference matrix is compatible with the rest of the information provided by the experts.

The paper is organized as follows. Some elements of Alo-groups are summarized in Section 2. In Section 3, preference matrices with elements from an Alo-grup are investigated, a reciprocity and consistency conditions are defined as well as inconsistency index of the P-matrix. The priority vector for ranking the alternatives is also defined. In Section 4, a special notation for the matrix with missing elements is introduced and the concept of the extension of P-matrix with missing elements is defined. This concept is based on a particular representation of consistent matrix and the missing elements of the extended matrix are calculated by applying the generalized least squares method. In Section 5, two special cases of P-matrix with missing elements are investigated. Here, for an $n \times n$ P-matrix the 
expert evaluates only $n-1$ pairs of alternatives. In this section, two numerical examples illustrating the necessary and sufficient conditions for elements to be evaluated in the P-matrix are presented. In Section 6 , some concluding considerations and remarks are presented.

\section{Abelian linearly ordered groups}

In this section, some elements of Abelian linearly ordered groups (Alo-groups) are summarized. The content of this section is based mainly on [7], or [3].

An Abelian group is a set, $\mathbf{G}$, together with an operation $\odot$ (read: operation odot) that combines any two elements $a, b \in \mathbf{G}$ to form another element denoted by $a \odot b$. The symbol $\odot$ is a general placeholder for a concretely given operation. The set and operation, $(\mathbf{G}, \odot)$, satisfies the following requirements known as the Abelian group axioms:

- If $a, b \in \mathbf{G}$, then $a \odot b \in \mathbf{G}$ (closure).

- If $a, b, c \in \mathbf{G}$, then $(a \odot b) \odot c=a \odot(b \odot c)$ (associativity).

- There exists an element $e \in \mathbf{G}$ called the identity element, such that for all $a \in \mathbf{G}, e \odot a=$ $a \odot e=a$ (identity element).

- If $a \in \mathbf{G}$, then there exists an element $a^{(-1)} \in$ $\mathbf{G}$ called the inverse element to $a$ such that $a \odot a^{(-1)}=a^{(-1)} \odot a=e$ (inverse element $)$.

- If $a, b \in \mathbf{G}$, then $a \odot b=b \odot a$ (commutativity).

The inverse operation $\div$ to $\odot$ is defined for all $a, b \in \mathbf{G}$ as follows: $a \div b=a \odot b^{(-1)}$.

A nonempty set $\mathbf{G}$ is linearly (totally) ordered under the order relation $\leq$, if the following statements hold for all $a, b, c \in \mathbf{G}$ :

- If $a \leq b$ and $b \leq a$, then $a=b$ (antisymmetry).

- If $a \leq b$ and $b \leq c$, then $a \leq c$ (transitivity).

- $a \leq b$ or $b \leq a$ (totality).

The strict order relation $<$ is defined for $a, b \in \mathbf{G}$ : $a<b$ if $a \leq b$ and $a \neq b$.

Let $(\mathbf{G}, \odot)$ be an Abelian group, $\mathbf{G}$ be linearly ordered under $\leq$. $(\mathbf{G}, \odot, \leq)$ is said to be an Abelian linearly ordered group, Alo-group for short, if for all $c \in \mathbf{G}: a \leq b$ implies $a \odot c \leq b \odot c$.

If $\mathcal{G}=(\mathbf{G}, \odot, \leq)$ is an Alo-group, then $\mathbf{G}$ is naturally equipped with the order topology induced by $\leq$ and $\mathbf{G} \times \mathbf{G}$ is equipped with the related product topology. We say that $\mathcal{G}$ is a continuous Alo-group if $\odot$ is continuous on $\mathbf{G} \times \mathbf{G}$.

Because of the associative property, the operation $\odot$ can be extended by induction to $n$-ary operation, $n>2$. Then, for a positive integer $n$, the $(n)$ power $a^{(n)}$ of $a \in \mathbf{G}$ is defined. We can extend the meaning of power $a^{(s)}$ to the case that $s$ is a negative integer.

$\mathcal{G}=(\mathbf{G}, \odot, \leq)$ is divisible if for each positive integer $n$ and each $a \in \mathbf{G}$ there exists the $(n)$-th root of $a$ denoted by $a^{(1 / n)}$, i.e. $\left(a^{(1 / n)}\right)^{(n)}=a$.
Moreover, the function $\|\|:. \mathbf{G} \rightarrow \mathbf{G}$ defined for each $a \in \mathbf{G}$ by $\|a\|=\max \left\{a, a^{(-1)}\right\}$ is called a $\mathcal{G}$-norm. The operation $d: \mathbf{G} \times \mathbf{G} \rightarrow \mathbf{G}$ defined by $d(a, b)=\|a \div b\|$ for all $a, b \in \mathbf{G}$ is called a $\mathcal{G}$-distance. It is easy to show that $d$ satisfies the usual distance properties.

Example 1 Additive Alo-group

$\mathcal{R}=(]-\infty,+\infty[,+, \leq)$ is a continuous Alo-group with: $e=0, a^{(-1)}=-a, a^{(n)}=n . a$.

Example 2 Multiplicative Alo-group

$\mathcal{R}^{+}=(] 0,+\infty[, \bullet, \leq)$ is a continuous Alo-group with: $e=1, a^{(-1)}=a^{-1}=1 / a, a^{(n)}=a^{n}$. Here, by symbol • the usual multiplication is denoted.

Example 3 Fuzzy additive Alo-group

$\mathcal{R}_{a}=(]-\infty,+\infty\left[,{ }_{f}, \leq\right)$, see [25], is a continuous Alo-group with: $a+{ }_{f} b=a+b-0.5, e=0.5, a^{(-1)}=$ $1-a, a^{(n)}=n . a-\frac{n-1}{2}$.

Example 4 Fuzzy multiplicative Alo-group ] $0,1\left[m=(] 0,1\left[, \bullet_{f}, \leq\right), \quad\right.$ is a continuous Alo-group with: $a \bullet_{f} b=\frac{a b}{a b+(1-a)(1-b)}, e=0.5, a^{(-1)}=$ $1-a, a^{(n)}=\frac{a^{n}}{a^{n}+(1-a)^{n}}$.

\section{P-matrix on Alo-groups over a real interval}

Let $\mathbf{G}$ be an open interval of the real line $\mathbf{R}$ and $\leq$ be the total order on $\mathbf{G}$ inherited from the usual order on $\mathbf{R}, \mathcal{G}=(\mathbf{G}, \odot, \leq)$ be a real Alo-group. We also assume that $\mathcal{G}$ is a divisible and continuous Alo-group. Then $\mathbf{G}$ is an open interval, see [7].

The DM problem can be formulated as follows. Let $X=\left\{x_{1}, x_{2}, \ldots, x_{n}\right\}$ be a finite set of alternatives. These alternatives have to be classified from best to worst, using the information given by a DM in the form of pairwise comparison matrix.

The preferences over the set of alternatives, $X$, may be represented in the following way. Assume that the preferences on $X$ are described by a preference relation on $X$ given by an $n \times n$ matrix $A=\left\{a_{i j}\right\}$, where $a_{i j} \in \mathbf{G}$ for all $i, j=1,2, \ldots, n$ indicates a preference intensity for alternative $x_{i}$ to that of $x_{j}$, i.e. it is interpreted as " $x_{i}$ is $a_{i j}$ times better than $x_{j}$ ". The elements of $A=\left\{a_{i j}\right\}$ satisfy the following reciprocity condition, see [7].

An $n \times n$ matrix $A=\left\{a_{i j}\right\}$ is $\odot$-reciprocal, if

$$
a_{i j} \odot a_{j i}=e \text { for all } i, j=1,2, \ldots, n,
$$

or, equivalently,

$$
a_{j i}=a_{i j}^{(-1)} \text { for all } i, j=1,2, \ldots, n \text {. }
$$

An $n \times n$ matrix $A=\left\{a_{i j}\right\}$ is $\odot$-consistent [7], if

$$
a_{i k}=a_{i j} \odot a_{j k} \text { for all } i, j, k=1,2, \ldots, n \text {. }
$$


Here, $a_{i i}=e$ for all $i=1,2, \ldots, n$, and also (3) implies $(1)$, i.e. an $\odot$-consistent matrix is $\odot$-reciprocal (however, not vice-versa).

The following result gives a characterization of $\odot$-consistent matrix as by the vectors of weights, see [7].

Proposition $1 A$ P-matrix $A=\left\{a_{i j}\right\}$ is $\odot$ consistent if and only if there exists a vector $w=$ $\left(w_{1}, w_{2}, \ldots, w_{n}\right), w_{i} \in \mathbf{G}$ such that

$$
w_{i} \div w_{j}=a_{i j} \text { for all } i, j=1,2, \ldots, n \text {. }
$$

If for some $i, j, k=1,2, \ldots, n$ (3) is not satisfied we say that P-matrix $A=\left\{a_{i j}\right\}$ is inconsistent.

The inconsistency of $A$ will be measured by the $\odot$-mean distance of the ratio matrix $W=\left\{w_{i} \div w_{j}\right\}$ to matrix $A=\left\{a_{i j}\right\}$.

Let $A=\left\{a_{i j}\right\}, w=\left(w_{1}, \ldots, w_{n}\right), w_{i} \in \mathbf{G}$ for all $i=1,2, \ldots, n$. Denote

$$
I_{\odot}(A, w)=\left(\bigodot_{1 \leq i<j \leq n}\left\|a_{i j} \div\left(w_{i} \div w_{j}\right)\right\|\right)^{(2 /(n(n-1)))} .
$$

Now, a concept of priority vector shall be defined. Consider the following optimization problem.

subject to

$$
I_{\odot}(A, w) \longrightarrow \min _{e}
$$

$$
\begin{aligned}
& \bigodot_{k=1}^{n} w_{k}=e, \\
& w_{i} \in \mathbf{G}, i=1,2, \ldots, n .
\end{aligned}
$$

If an optimal solution of (P1) exists, then the $\odot$ consistency index of $A, I_{\odot}(A)$, is defined as

$$
I_{\odot}(A)=I_{\odot}\left(A, w^{*}\right)
$$

where $w^{*}=\left(w_{1}^{*}, \ldots, w_{n}^{*}\right)$ is the optimal solution of (P1). Notice that "minimization" in (P1) is carried out with respect to the identity element $e$.

An optimal solution $w^{*}$ of $(\mathrm{P} 1)$ is called the $\odot$-priority vector of $A$. In $(\mathrm{P} 1), \bigodot_{k=1}^{n} w_{k}=e$, is a normalization condition reducing the number of the priority vectors (uniqueness), on condition that the optimal solution exists. The proof of the following theorem is evident and it is left to the reader.

Proposition $2 A$ P-matrix $A=\left\{a_{i j}\right\}$ is $\odot$ consistent if and only if

$$
I_{\odot}(A)=e .
$$

\section{P-matrix with missing elements}

Usually, in many decision-making procedures, experts are capable of providing preference degrees between any pair of given alternatives. However, this may not be always true. A missing value can be the result of the incapacity of an expert to quantify the degree of preference of one alternative over another. In this case he/she may decide not to guess the preference degree between some pairs of alternatives. When an expert is not able to express a particular value $a_{i j}$, because he/she does not have a clear idea of how the alternative $x_{i}$ is better than alternative $x_{j}$, this does not mean that he/she prefers both options with the same intensity. In order to model these situations, in the following we introduce the incomplete preference matrix. Here, we use a different approach and notation compared to e.g. [1]. On the other hand, our approach is similar to that of [24].

Now, define the P-matrix with missing elements. For the sake of simplicity of presentation the alternatives $x_{1}, x_{2}, \ldots, x_{n}$ are identified with integers $1,2, \ldots, n$, i.e. by $X=\{1,2, \ldots, n\}$ we denote the set of alternatives, $n>1$. Moreover, let $X \times X=X^{2}$ be the Cartesian product of $X$, i.e. $X^{2}=\{(i, j) \mid i, j \in$ $X\}$. Let $K \subset X^{2}, K \neq X^{2}$ and $\mathcal{A}$ be the preference relation on $K$ given by the (membership) function $\mu_{\mathcal{A}}: K \rightarrow \mathbf{G}, \mathbf{G}$ is an Alo-group. The preference relation $\mathcal{A}$ is represented by the $n \times n$ preference matrix $A(K)=\left\{a_{i j}\right\}_{K}$ with missing elements depending on $K$ as follows

$$
a_{i j}= \begin{cases}\mu_{\mathcal{A}}(i, j) & \text { if } \quad(i, j) \in K \\ \times & \text { if } \quad(i, j) \notin K .\end{cases}
$$

In what follows we shall assume that each $\mathrm{P}$ matrix $A(K)=\left\{a_{i j}\right\}_{K}$ with missing elements is $\odot$-reciprocal, i.e.

$$
a_{i j} \odot a_{j i}=e \text { for all }(i, j) \in K .
$$

If $L \subset K$, and $L=\left\{\left(i_{1}, j_{1}\right),\left(i_{2}, j_{2}\right), \ldots,\left(i_{q}, j_{q}\right)\right\}$ is a set of couples $(i, j)$ of alternatives such that there exist $a_{i j}$, with $a_{i j} \in \mathbf{G}$ for all $(i, j) \in L$, then the symmetric subset $L^{\prime}$ to $L$, i.e. $L^{\prime}=$ $\left\{\left(j_{1}, i_{1}\right),\left(j_{2}, i_{2}\right), \ldots,\left(j_{q}, i_{q}\right)\right\}$ is also a subset of $K$, i.e. $L^{\prime} \subset K$. By reciprocity each subset $K$ of $X^{2}$ can be represented as follows: $K=L \cup L^{\prime} \cup D$, where $L$ is the set of couples of alternatives $(i, j)$ of given preference degrees $a_{i j}$ of the P-matrix $A(K)$ and $D$ is the diagonal of this matrix, i.e. $D=\{(1,1),(2,2), \ldots,(n, n)\}$, where $a_{i i}=e$ for all $i \in X$. The reciprocity property means that the expert is able to quantify both $a_{i j}$ and $a_{j i}$ as well as $a_{i i}$. The elements $a_{i j}$ with $(i, j) \in X^{2}-K$ are called the missing elements of matrix $A(K)$. Notice that the missing elements of $A(K)$ are denoted by symbol $\times(" \mathrm{ex} ")$. On the other hand, the elements - preference degrees given by the experts are denoted by $a_{i j}$ where $(i, j) \in K$. By $\odot$-reciprocity it is sufficient that in reality the expert will quantify only the elements $a_{i j}$, where $(i, j) \in L$, such that $K=L \cup L^{\prime} \cup D$. In what follows we shall investigate two important situations of $L$, particularly, $L=\{(1,2),(2,3), \ldots,(n-1, n)\}$, and $L=$ $\{(1,2),(1,3), \ldots,(1, n)\}$.

Now, we shall deal with the problem of finding the values of missing elements of a given $\mathrm{P}$-matrix so that the extended matrix is as much $\odot$-consistent 
as possible. In the ideal case the extended matrix would become $\odot$-consistent.

Let $K \subset X^{2}$, let $A(K)=\left\{a_{i j}\right\}_{K}$ be a Pmatrix with missing elements. The matrix $A^{e}(K)=$ $\left\{a_{i j}^{e}\right\}_{K}$ called the $\odot$-extension of $A(K)$ is defined as follows

$$
a_{i j}^{e}= \begin{cases}a_{i j} & \text { if } \quad(i, j) \in K, \\ v_{i}^{*} \div v_{j}^{*} & \text { if } \quad(i, j) \notin K .\end{cases}
$$

Here, $v^{*}=\left(v_{1}^{*}, v_{2}^{*}, \ldots, v_{n}^{*}\right)$ is called the $\odot$-priority vector with respect to $K$, if it is an optimal solution of the following optimization problem subject to

$$
d(v, K) \longrightarrow \min _{e}
$$

$$
\begin{aligned}
& \bigodot_{j=1}^{n} v_{j}=e, \\
& v_{i} \in \mathbf{G} \text { for all } i=1,2, \ldots, n .
\end{aligned}
$$

Here, $d(v, K)=\left(\bigodot_{i, j \in K}\left\|a_{i j} \div\left(v_{i} \div v_{j}\right)\right\|\right)^{(1 /|K|)}$, $|K|$ denotes the cardinality of $K$. Notice, that $\odot-$ consistency index of the matrix $A^{e}(K)=\left\{a_{i j}^{e}\right\}_{K}$ is defined by (6) as $I_{\odot}\left(A^{e}(K)\right)$. Minimization in (P2) is carried out with respect to the identity element $e$.

The proof of the following proposition follows directly from Proposition 2.

Proposition $3 A^{e}(K)=\left\{a_{i j}^{e}\right\}_{K}$ is $\odot$-consistent, (i.e. $I_{\odot}\left(A^{e}(K)\right)=e$ ) if and only if

$$
d\left(v^{*}, K\right)=e
$$

\section{Special cases of preference matrix with missing elements}

For a complete reciprocal preference $n \times n$ matrix we need $N=\frac{n(n-1)}{2}$ pairs of elements to be evaluated by an expert. For example, if $n=12$, then $N=66$, which is a considerable number of pairwise comparisons. We ask that the expert would evaluate only "around $n$ "pairwise comparisons of alternatives which seems to be a reasonable amount. In this section we shall investigate two important particular cases of fuzzy preference matrix with missing elements where the expert will evaluate only $n-1$ pairwise comparisons of alternatives. Here, the approach presented in [24] is generalized. Let $K \subset X^{2}$ be a set of indices given by an expert, $A(K)=\left\{a_{i j}\right\}_{K}$ be a P-matrix with missing elements. Moreover, let $K=L \cup L^{\prime} \cup D$. In fact, it is sufficient to assume that the expert will evaluate only a chain of matrix elements of $L$, i.e. $a_{12}, a_{23}, a_{34}, \ldots, a_{n-1, n}$.

\subsection{Case $L=\{(1,2),(2,3), \ldots,(n-1, n)\}$}

Here, assume that the expert evaluates $n-1$ chain elements of the P-matrix $A(K)$, i.e. $a_{12}, a_{23}, a_{34}, \ldots, a_{n-1, n}$. $\odot$-extension of $A(K)$. result.

Proposition 4 Let $L=\{(1,2),(2,3), \ldots,(n-$ $1, n)\}, a_{i j} \in \mathbf{G}$ with $a_{i j} \odot a_{j i}=e$ for all $(i, j) \in K$, $K=L \cup L^{\prime} \cup D$, and $L^{\prime}=\{(2,1),(3,2), \ldots,(n, n-$ $1)\}, D=\{(1,1), \ldots,(n, n)\}$. Then $\odot$-priority vector $v^{*}=\left(v_{1}^{*}, v_{2}^{*}, \ldots, v_{n}^{*}\right)$ with respect to $K$ is given as

$$
\begin{aligned}
& v_{1}^{*}=\left(\bigodot_{i=2}^{n}\left(a_{12} \odot \ldots \odot a_{i-1, i}\right)\right)^{(1 / n)}, \\
& v_{i}^{*}=a_{i-1, i}^{(-1)} \odot v_{i-1}^{*} \text { for } i=2,3, \ldots, n .
\end{aligned}
$$

Proof.

If (7) and (8) are satisfied, then

$v_{i}^{*}=a_{i-1, i} \odot a_{i-2, i-1} \odot \ldots \odot a_{1,2} \odot v_{1}^{*}$ for $i=2, \ldots, n$, hence for all $i=1,2, \ldots, n, v_{i}^{*} \in \mathbf{G}$ and

$$
\bigodot_{i=1}^{n} v_{i}^{*}=e \text {. }
$$

Also,

$$
a_{i-1, i}=v_{i-1}^{*} \div v_{i}^{*} \text { for } i=2, \ldots, n .
$$

Then $v=\left(v_{1}^{*}, \ldots, v_{1}^{*}\right)$ is an optimal solution of $(\mathrm{P} 2)$.

As a simple consequence, we obtain the following corollary.

Corollary 5 Let $\mathcal{R}=(]-\infty,+\infty[,+, \leq)$ be an additive Alo-group, see Example 1, i.e. $\odot=+$. Then we obtain (7), (8) in the following form:

$$
\begin{gathered}
v_{1}^{*}=\frac{1}{n} \sum_{i=2}^{n}(n-i+1) a_{i-1, i}, \\
v_{i}^{*}=v_{i-1}^{*}-a_{i-1, i} \text { for } i=2,3, \ldots, n .
\end{gathered}
$$

Example 5 Let $\odot=+, L=\{(1,2),(2,3),(3,4)\}$, see Example 1. Let the chain evaluations be $a_{12}=$ $9, a_{23}=8, a_{34}=5$, with $a_{i j}+a_{j i}=0$ for all $(i, j) \in$ $L, K=L \cup L^{\prime} \cup D$. Hence $A(K)=\left\{a_{i j}\right\}_{K}$ is a $P$-matrix with missing elements as follows

$$
A(K)=\left(\begin{array}{cccc}
0 & 9 & \times & \times \\
-9 & 0 & 8 & \times \\
\times & -8 & 0 & 5 \\
\times & \times & -5 & 0
\end{array}\right) .
$$

By (9), (10) we obtain +-priority vector $v^{*}$ with respect to $K$, particularly, $v^{*}=(12,3,-5,-10)$. By (4) we obtain $A^{e}(K)$ - +-extension of $A(K)$ as follows

$$
A^{e}(K)=\left(\begin{array}{cccc}
0 & 9 & 17 & 22 \\
-9 & 0 & 8 & 13 \\
-17 & -8 & 0 & 5 \\
-22 & -13 & -5 & 0
\end{array}\right)
$$

where, $A^{e}(K)$ is +-consistent, and $d(v, B(K))=0$, hence $I_{+}\left(A^{e}(K)\right)=0$. The corresponding ranking of the alternatives is $x_{1}>x_{2}>x_{3}>x_{4}$. 
Also, as a simple consequence, the following corollary is obtained.

Corollary 6 Let $\mathcal{R}^{+}=(] 0,+\infty[, \bullet, \leq)$ be a multiplicative Alo-group, see Example 2, i.e. $\odot=\bullet$. Then we obtain (7), (8) in the following form:

$$
\begin{gathered}
P_{1}=1, P_{i}=P_{i-1} a_{i-1, i}, \text { for } i=2,3, \ldots, n, \\
v_{1}^{*}=\left(\prod_{i=1}^{n} P_{i}\right)^{\frac{1}{n}} \\
v_{i}^{*}=\frac{v_{i-1}^{*}}{a_{i-1, i}} \text { for } i=2,3, \ldots, n .
\end{gathered}
$$

Example 6 Let $\odot=\bullet, L=\{(1,2),(2,3),(3,4)\}$, see Example 2. Let the chain evaluations be $a_{12}=$ $4, a_{23}=3, a_{34}=2$, with $a_{i j} \bullet a_{j i}=1$ for all $(i, j) \in$ $L, K=L \cup L^{\prime} \cup D$. Hence $A(K)=\left\{a_{i j}\right\}_{K}$ is a $P$-matrix with missing elements as follows

$$
A(K)=\left(\begin{array}{cccc}
1 & 4 & \times & \times \\
\frac{1}{4} & 1 & 3 & \times \\
\times & \frac{1}{3} & 1 & 2 \\
\times & \times & \frac{1}{2} & 1
\end{array}\right)
$$

By (11), (12), (13) we obtain •-priority vector $v^{*}$ with respect to $K$, particularly, $v^{*}=$ $(5.826,1.456,0.485,0.243)$. By (4) we obtain $A^{e}(K)$

- •-extension of $A(K)$ as follows

$$
A^{e}(K)=\left(\begin{array}{cccc}
1 & 4 & 12 & 24 \\
\frac{1}{4} & 1 & 3 & 6 \\
\frac{1}{12} & \frac{1}{3} & 1 & 2 \\
\frac{1}{24} & \frac{1}{6} & \frac{1}{2} & 1
\end{array}\right)
$$

where, $A^{e}(K)$ is •-consistent, and $d(v, B(K))=1$, hence $I_{\bullet}\left(A^{e}(K)\right)=1$. The corresponding ranking of the alternatives is $x_{1}>x_{2}>x_{3}>x_{4}$.

Corollary 7 Let $\mathcal{R}_{a}=(]-\infty,+\infty\left[,+_{f}, \leq\right)$ be a fuzzy additive Alo-group, see Example 3, i.e. $\odot=$ $+_{f}$. Then we obtain (7), (8) in the following form:

$$
\begin{gathered}
S_{1}=0, S_{i}=S_{i-1}+a_{i-1, i}, \text { for } i=2,3, \ldots, n, \\
v_{1}^{*}=\frac{3-n}{4}+\frac{1}{n} \sum_{i=1}^{n} S_{i}, \\
v_{i}^{*}=v_{i-1}^{*}-a_{i-1, i}+0.5 \text { for } i=2,3, \ldots, n .
\end{gathered}
$$

Example 7 Let $\odot=+_{f}, L=\{(1,2),(2,3),(3,4)\}$, see Example 3. Let the chain evaluations be $a_{12}=$ $0.9, a_{23}=0.5, a_{34}=0.3$, with $a_{i j}+{ }_{f} a_{j i}=0.5$ for all $(i, j) \in L, K=L \cup L^{\prime} \cup D$. Hence $A(K)=\left\{a_{i j}\right\}_{K}$ is a P-matrix with missing elements as follows

$$
A(K)=\left(\begin{array}{cccc}
0.5 & 0.9 & \times & \times \\
0.1 & 0.5 & 0.5 & \times \\
\times & 0.5 & 0.5 & 0.3 \\
\times & \times & 0.7 & 0.5
\end{array}\right)
$$

By (14), (15), (16) we obtain $+_{f}$-priority vector $v^{*}$ with respect to $K$, particularly, $v^{*}=$
$(0.75,0.35,0.35,0.55)$. By (4) we obtain $A^{e}(K)$ ${ }{ }_{f}$-extension of $A(K)$ as follows

$$
A^{e}(K)=\left(\begin{array}{cccc}
0.5 & 0.9 & 0.9 & 0.7 \\
0.1 & 0.5 & 0.5 & 0.3 \\
0.1 & 0.5 & 0.5 & 0.3 \\
0.3 & 0.7 & 0.7 & 0.5
\end{array}\right)
$$

where, $A^{e}(K)$ is $+_{f}$-consistent, and $d(v, B(K))=$ 0.5 , hence $I_{+_{f}}\left(A^{e}(K)\right)=0.5$. The corresponding ranking of the alternatives is $x_{1}>x_{4}>x_{2} \sim x_{3}$. Here, by the symbol $\sim$ the same order of $x_{2}$ and $x_{3}$ is denoted.

We obtain also the following corollary.

Corollary 8 Let $] 0,1\left[_{m}=(] 0,1\left[, \bullet_{f}, \leq\right)\right.$ be a fuzzy multiplicative Alo-group, see Example 4, i.e. $\odot=$ - $f$. Then for $i=2,3, \ldots, n$, we obtain (7), (8) in the following form:

$$
\begin{gathered}
P_{i}=\frac{\left(1-a_{12}\right) \cdot \ldots \cdot\left(1-a_{i-1, i}\right)}{\left(1-a_{12}\right) \cdot \ldots \cdot\left(1-a_{i-1, i}\right)+a_{12} \cdot \ldots \cdot a_{i-1, i}}, \\
P=\frac{P_{1} \cdot \ldots \cdot P_{n}}{\left(1-P_{1}\right) \cdot \ldots \cdot\left(1-P_{n}\right)+P_{1} \cdot \ldots \cdot P_{n}}, \\
v_{i}^{*}=\frac{(1-P)^{1 / n}}{(1-P)^{1 / n}+P^{1 / n}} \\
\left(1-a_{i-1, i}\right) v_{i-1}^{*}+a_{i-1, i}\left(1-v_{i-1}^{*}\right)
\end{gathered}
$$

Formulas (17), (18), (19) and (20) can be easily calculated e.g. by Excel.

Example 8 Let $\odot=\bullet_{f}, L=\{(1,2),(2,3),(3,4)\}$, see Example 4. Let the chain evaluations be $a_{12}=$ $0.9, a_{23}=0.5, a_{34}=0.3$, with $a_{i j} \bullet_{f} a_{j i}=0.5$ for all $(i, j) \in L, K=L \cup L^{\prime} \cup D$. Hence $A(K)=\left\{a_{i j}\right\}_{K}$ is a P-matrix with missing elements as follows

$$
A(K)=\left(\begin{array}{cccc}
0.5 & 0.9 & \times & \times \\
0.1 & 0.5 & 0.5 & \times \\
\times & 0.5 & 0.5 & 0.3 \\
\times & \times & 0.7 & 0.5
\end{array}\right)
$$

By (18), (19) we obtain •-priority vector $v^{*}$ with respect to $K$, particularly, $v^{*}=(0.808,0.318,0.318,0.522) . \quad$ By (4) we obtain $A^{e}(K)-\bullet_{f}$-extension of $A(K)$ as follows

$$
A^{e}(K)=\left(\begin{array}{cccc}
0.5 & 0.9 & 0.9 & 0794 \\
0.1 & 0.5 & 0.5 & 0.3 \\
0.1 & 0.5 & 0.5 & 0.3 \\
0.206 & 0.7 & 0.7 & 0.5
\end{array}\right)
$$

where, $A^{e}(K)$ is -consistent, and $d(v, B(K))=$ 0.5 , hence $I_{\bullet}\left(A^{e}(K)\right)=0.5$. The corresponding ranking of the alternatives is $x_{1}>x_{4}>x_{2} \sim x_{3}$. 


\subsection{Case $L=\{(1,2),(1,3), \ldots,(1, n)\}$}

Now, assume that the expert evaluates the pairs of a given fixed element with the remaining $n-1$ elements, i.e. the P-matrix $A(K)$ is given by $a_{12}, a_{13}, \ldots, a_{1 n}$. We investigate the extension of $A(K)$ and obtain the following result.

Proposition 9 Let $L=\{(1,2),(1,3), \ldots,(1, n)\}$, $a_{i j} \in \mathbf{G}$ with $a_{i j} \odot a_{j i}=e$ for all $(i, j) \in K, K=$ $L \cup L^{\prime} \cup D$, and $L^{\prime}=\{(2,1),(3,1), \ldots,(n, 1)\}, D=$ $\{(1,1), \ldots,(n, n)\}$. Then $\odot$-priority vector $v^{*}$ $=\left(v_{1}^{*}, v_{2}^{*}, \ldots, v_{n}^{*}\right)$ with respect to $K$ is given as

$$
\begin{gathered}
v_{1}^{*}=\left(\bigodot_{i=2}^{n} a_{1 i}\right)^{(1 / n)}, \\
v_{i}^{*}=a_{1, i}^{(-1)} \odot v_{1}^{*} \text { for } i=2,3, \ldots, n .
\end{gathered}
$$

Proof.

If (21) and (22) are satisfied, then

$v_{i}^{*}=a_{1, i-1} \odot a_{1, i-2} \odot \ldots \odot a_{1,2} \odot v_{1}^{*}$ for $i=2, \ldots, n$, hence for all $i=1,2, \ldots, n, v_{i}^{*} \in \mathbf{G}$, moreover,

$$
\bigodot_{i=1}^{n} v_{i}^{*}=e
$$

and also

$$
a_{1, i-1}=v_{1}^{*} \div v_{i}^{*} \text { for } i=2, \ldots, n .
$$

Then $v=\left(v_{1}^{*}, \ldots, v_{1}^{*}\right)$ is an optimal solution of $(\mathrm{P} 2)$.

As a simple consequence, we obtain the following corollary.

Corollary 10 Let $\mathcal{R}=(]-\infty,+\infty[,+, \leq)$ be an additive Alo-group, see Example 1, i.e. $\odot=+$. Then we obtain (21), (22) in the following form

$$
\begin{gathered}
v_{1}^{*}=\frac{1}{n} \sum_{i=2}^{n} a_{1, i}, \\
v_{i}^{*}=v_{1}^{*}-a_{1, i} \text { for } i=2,3, \ldots, n .
\end{gathered}
$$

Moreover, the extension of $A(K)$, i.e. matrix $A^{e}(K)=\left\{a_{i j}^{a c}\right\}_{K}$ is $\odot$-consistent.

Example $9 \odot=+, L=\{(1,2),(1,3),(1,4)\}$, let the expert evaluations be $b_{12}=9, b_{13}=8, b_{14}=5$, with $b_{i j}+b_{j i}=0$ for all $(i, j) \in L$, let $K=L \cup L^{\prime} \cup D$. Let $B(K)=\left\{b_{i j}\right\}_{K}$ be a P- matrix with missing elements as follows

$$
B(K)=\left(\begin{array}{cccc}
0 & 9 & 8 & 5 \\
-9 & 0 & \times & \times \\
-8 & \times & 0 & \times \\
-5 & \times & \times & 0
\end{array}\right)
$$

By (23), (24) we obtain +-priority vector $w^{*}$ with respect to $K$, particularly, $w^{*}=(5.5,-3.5,-2.5,0.5) . \quad B y$ (4) we obtain $B^{e}(K)$ - +-extension of $B(K)$ as follows

$$
B^{e}(K)=\left(\begin{array}{cccc}
0 & 9 & 8 & 5 \\
-9 & 0 & -1 & -4 \\
-8 & 1 & 0 & -3 \\
-5 & 4 & 3 & 0
\end{array}\right)
$$

where, $B^{e}(K)$ is +-consistent, and $d(v, B(K))=0$, hence $I_{+}\left(B^{e}(K)\right)=0$. The corresponding ranking of the alternatives is $x_{1}>x_{4}>x_{3}>x_{2}$.

Corollary 11 Let $\mathcal{R}^{+}=(] 0,+\infty[, \bullet, \leq)$ be a multiplicative Alo-group, see Example 2, i.e. $\odot=\bullet$. Then we obtain (21), (22) in the following form

$$
\begin{gathered}
v_{1}^{*}=\left(\prod_{i=2}^{n} a_{1, i}\right)^{1 / n}, \\
v_{i}^{*}=\frac{v_{1}^{*}}{a_{1, i}} \text { for } i=2,3, \ldots, n .
\end{gathered}
$$

Moreover, the extension of $A(K)$, i.e. matrix $A^{e}(K)=\left\{a_{i j}^{a c}\right\}_{K}$ is •-consistent.

Example $10 \odot=\bullet, L=\{(1,2),(1,3),(1,4)\}$, see Example 2. Let the expert evaluations be $b_{12}=$ $4, b_{13}=3, b_{14}=2$, with $b_{i j} \bullet b_{j i}=1$ for all $(i, j) \in L$, let $K=L \cup L^{\prime} \cup D$. Let $B(K)=\left\{b_{i j}\right\}_{K}$ be a $P$ matrix with missing elements as follows

$$
B(K)=\left(\begin{array}{cccc}
1 & 4 & 3 & 2 \\
\frac{1}{4} & 1 & \times & \times \\
\frac{1}{3} & \times & 1 & \times \\
\frac{1}{2} & \times & \times & 1
\end{array}\right) .
$$

By (25), (26) we obtain •-priority vector $w^{*}$ with respect to $K$, particularly, $w^{*}=(2.213,0.553,0.738,1.107) . \quad B y$ (4) we obtain $B^{e}(K)$ - •-extension of $B(K)$ as follows

$$
B^{e}(K)=\left(\begin{array}{cccc}
1 & 4 & 3 & 2 \\
\frac{1}{4} & 1 & \frac{3}{4} & \frac{1}{2} \\
\frac{1}{3} & \frac{4}{3} & 1 & \frac{2}{3} \\
\frac{1}{2} & 2 & \frac{3}{2} & 1
\end{array}\right)
$$

where, $B^{e}(K)$ is $\bullet$-consistent, and $d(v, B(K))=1$, hence $I_{\bullet}\left(B^{e}(K)\right)=1$. The corresponding ranking of the alternatives is $x_{1}>x_{2} \sim x_{3}>x_{4}$.

Corollary 12 Let $\mathcal{R}_{a}=(]-\infty,+\infty\left[,{ }_{f}, \leq\right)$ be a fuzzy additive Alo-group , see Example 3, i.e. $\odot=$ $+_{f}$. Then we obtain (21), (22) in the following form

$$
\begin{gathered}
v_{1}^{*}=\frac{1}{2 n}+\frac{1}{n} \sum_{i=2}^{n} a_{1, i}, \\
v_{i}^{*}=v_{1}^{*}-a_{1, i}+0.5 \text {. for } i=2,3, \ldots, n .
\end{gathered}
$$

Moreover, the extension of $A(K)$, i.e. matrix $A^{e}(K)=\left\{a_{i j}^{a c}\right\}_{K}$ is $+_{f}$-consistent. 
Example $11 \odot=+_{f}, L=\{(1,2),(1,3),(1,4)\}$, let the expert evaluations be $b_{12}=0.9, b_{13}=$ $0.5, b_{14}=0.3$, with $b_{i j}+{ }_{f} b_{j i}=0.5$ for all $(i, j) \in L$, let $K=L \cup L^{\prime} \cup D$. Let $B(K)=\left\{b_{i j}\right\}_{K}$ be a Pmatrix with missing elements as follows

$$
B(K)=\left(\begin{array}{cccc}
0.5 & 0.9 & 0.6 & 0.4 \\
0.1 & 0.5 & \times & \times \\
0.4 & \times & 0.5 & \times \\
0.6 & \times & \times & 0.5
\end{array}\right)
$$

By (27), (28) we obtain $+_{f}$-priority vector $w^{*}$ with respect to $K$, particularly, $w^{*}=(0.6,0,2,0.5,0.7)$. By (4) we obtain $B^{e}(K)-+_{f}$-extension of $B(K)$ as follows

$$
B^{e}(K)=\left(\begin{array}{cccc}
0.5 & 0.9 & 0.6 & 0.4 \\
0.1 & 0.5 & 0.2 & 0.0 \\
0.4 & 0.8 & 0.5 & 0.3 \\
0.6 & 1.0 & 0.7 & 0.5
\end{array}\right)
$$

where, $B^{e}(K)$ is $+_{f}$-consistent, and $d(v, B(K))=$ 0.5 , hence $I_{+_{f}}\left(B^{e}(K)\right)=0.5$. The corresponding ranking of the alternatives is $x_{4}>x_{1}>x_{3}>x_{2}$.

Corollary 13 Let $] 0,1\left[{ }_{m}=(] 0,1\left[, \bullet_{f}, \leq\right)\right.$ be a fuzzy multiplicative Alo-group , see Example 3, i.e. $\odot=$ $\bullet_{f}$. Then for $i=2,3, \ldots, n$ we obtain (21), (22) in the following form:

$$
\begin{gathered}
P_{i}=\frac{a_{1, i}^{1 / n}}{a_{1, i}^{1 / n}+\left(1-a_{1, i}\right)^{1 / n}} \\
v_{1}^{*}=\frac{P_{1} \cdot \ldots \cdot P_{n}}{P_{1} \cdot \ldots \cdot P_{n}+\left(1-P_{1}\right) \cdot \ldots \cdot\left(1-P_{n}\right)}, \\
v_{i}^{*}=\frac{\left(1-a_{1, i}\right) v_{1}^{*}}{\left(1-a_{1, i}\right) v_{1}^{*}+a_{1, i}\left(1-v_{1}^{*}\right)} .
\end{gathered}
$$

Moreover, the extension of $A(K)$, i.e. matrix $A^{e}(K)=\left\{a_{i j}^{a c}\right\}_{K}$ is $\bullet_{f}$-consistent.

Example $12 \odot=\bullet_{f}, L=\{(1,2),(1,3),(1,4)\}$, $b_{12}=0.9, b_{13}=0.6, b_{14}=0.4$, with $b_{i j} \bullet_{f} b_{j i}=0.5$ for all $(i, j) \in L$, let $K=L \cup L^{\prime} \cup D$. Let $B(K)=\left\{b_{i j}\right\}_{K}$ be a $P$ - matrix with missing elements as follows (see Example 4 and 10):

$$
B(K)=\left(\begin{array}{cccc}
0.5 & 0.9 & 0.6 & 0.4 \\
0.1 & 0.5 & \times & \times \\
0.4 & \times & 0.5 & \times \\
0.6 & \times & \times & 0.5
\end{array}\right)
$$

By (29), (30), (31) we obtain $\bullet_{f}$-priority vector $w^{*}$ with respect to $K$, particularly, $w^{*}=$ $(0.634,0.161,0.536,0.722)$. By (4) we obtain $B^{e}(K)$ - +-extension of $B(K)$ as follows

$$
B^{e}(K)=\left(\begin{array}{cccc}
0.5 & 0.9 & 0.6 & 0.4 \\
0.1 & 0.5 & 0.143 & 0.069 \\
0.4 & 0.857 & 0.5 & 0.308 \\
0.6 & 0.931 & 0.692 & 0.5
\end{array}\right)
$$

where, $B^{e}(K)$ is $\bullet_{f}$-consistent, and $d(v, B(K))=$ 0.5 , hence $I_{\bullet_{f}}\left(B^{e}(K)\right)=0.5$. The corresponding ranking of the alternatives is $x_{4}>x_{1}>x_{3}>x_{2}$.

\section{Conclusions}

In this paper we have dealt with some properties of P-matrices, particularly reciprocity and consistency, with the entries from the Alo-group. We have shown how to measure the grade of consistency and also how to evaluate the pairs of elements by values taken from the Alo-group if some elements are missing. Moreover, we have dealt with two particular cases of the incomplete P-matrix, and we have proposed some special methods for dealing with such cases. Finally, eight numerical examples have been presented to clarify our approach.

\section{References}

[1] Alonso, S., Chiclana, F., Herrera. F., HerreraViedma, E., Alcala-Fdes, J., Porcel, C. A consistency-based procedure to estimate missing pairwise preference values, Internat. J. Intelligent Syst., 23: 155-175, 2008.

[2] Barzilai, J. Consistency Measures for Pairwise Comparison Matrices, J. Multi-Crit. Decis. Anal., 7:123-132, 1998.

[3] Bourbaki, N. Algebra II, Springer Verlag, Heidelberg-New York-Berlin, 1998.

[4] Brin, S., Page. L. The anatomy of a large-scale hypertextualweb search engine, Computer Networks and ISDN Systems, 30:107-117, 1998.

[5] Buckley, J.J. Fuzzy hierarchical analysis, Fuzzy Sets and Systems, 17, 1:233-247, 1985.

[6] Cavallo, B., D'Apuzzo, L. A general unified framework for pairwise comparison matrices in multicriteria methods, International Journal of Intelligent Systems, 24, 4:377-398, 2009.

[7] Cavallo, B., D'Apuzzo, L., Squillante, M. About a Consistency Index for Pairwise Comparison Matrices over a Divisible Alo-Group, International J. of Intelligent Systems, 27:153-175, 2012.

[8] Chiclana, F. Herrera, F., Herrera-Viedma, E. Integrating Multiplicative Preference Relations in a Multipurpose Decision Making Model Based on Fuzzy Preference Relations, Fuzzy Sets and Systems, 112:277-291, 1991.

[9] Chiclana, F. Herrera, F., Herrera-Viedma, E., Alonso, S. Some induced ordered weighted averaging operators and their use for solving group decision-making problems based on fuzzy preference relations, European Journal of Operat. Res., 182:383-399, 2007.

[10] Chiclana, F., Herrera-Viedma, E., Alonso, S. A note on two methods for estimating pairwise preference values, IEE Transactions Syst. Man and cybernetics, 39, 6:1628-1633, 2009. 
[11] Fiedler, M., Nedoma, J., Ramík, J., Rohn, J., Zimmermann, K. Linear Optimization Problems with Inexact Data, Springer, Berlin - Heidelberg - New York - Hong Kong - London - Milan, Tokyo, 2006.

[12] Fodor, J., Roubens, M. Fuzzy Preference Modelling and Multicriteria Decision Support, Kluwer, Dordrecht, 1994.

[13] Herrera-Viedma, E., Herrera, F., Chiclana, F., Luque, M. Some issues on consistency of fuzzy preference relations, European Journal of $\mathrm{Op}$ erat. Res., 154: 98-109, 2004.

[14] Herrera, F., Herrera-Viedma, E., Chiclana, F. Multiperson decision making based on multiplicative preference relations, European J. Operat. Res., 129:372-385, 2001.

[15] Herrera, F., Herrera-Viedma, E. Choice functions and mechanisms for linguistic preference relations, European J. Operat. Res., 120:144$161,2000$.

[16] Herrera-Viedma, E., Chiclana, F., Herrera, F., Alonso, S. A group decision-making model with incomplete fuzzy preference relations based on additive consistency, IEEE Trans. Syst, Man Cybernetics, Part B, 37, 1:176-189, 2007.

[17] Kim, S. H, Choi, S. H, Kim, J. K. An interactive procedure for multiple attribute group decision making with incomplete information: Range-based approach, European J. Operat. Res., 118:139-152, 1999.

[18] Ma, J. et al. A method for repairing the inconsistency of fuzzy preference relations, Fuzzy Sets and Systems, 157:20-33, 2006.

[19] Mikhailov, L. Deriving priorities from fuzzy pairwise comparison judgments, Fuzzy Sets and Systems, 134:365-385, 2003.

[20] Ohnishi, S., Dubois, D. at al. A fuzzy constraint based approach to the AHP, In Uncertainty and Intelligent Inf. Syst., World Sci., Singapore, 217-228, 2008.

[21] Ramík, J., Vlach, M. Measuring consistency and inconsistency of pair comparison systems, Kybernetika, 49, 3:465-486, 2013.

[22] Ramík, J., Korviny, P. Inconsistency of pairwise comparison matrix with fuzzy elements based on geo-metric mean, Fuzzy Sets and Systems, 161:1604-1613, 2010.

[23] Ramík, J., Vlach, M. Generalized concavity in optimization and decision making, Kluwer Publ. Comp., Boston-Dordrecht-London, 2001.

[24] Ramik, J. Incomplete fuzzy preference matrix and its application to ranking of alternatives, Internat J. of Intelligent Systems, 28, 8:787-806, 2014.

[25] Gavalec, M., Ramik, J., Zimmermann, K. Decision making and Optimization: Special Matrices and Their Applications in Economics and Management, Springer Internat. Publ. Switzerland, Cham-Heidelberg-New York-DordrechtLondon, 2015.
[26] Saaty, T. L. The analytic hierarchy process, McGraw-Hill, New York, 1980.

[27] Saaty, T. L.: Fundamentals of Decision Making and Priority Theory with the AHP, RWS Publications, Pittsburgh, 1994.

[28] Switalski, Z.: General transitivity conditions for fuzzy reciprocal preference matrices, Fuzzy Sets and Systems,137 (2003), 85-100.

[29] Tanino, T. Fuzzy Preference Orderings in Group Decision Making, Fuzzy Sets and Systems, 12:117-131, 1984.

[30] Tanino, T. Fuzzy Preference Relations in Group Decision Making. In J. Kacprzyk, M. Roubens (Eds.), Non-Conventional Preference Relations in Decision Making, Springer-Verlag, Berlin, 54-71, 1988.

[31] Van Laarhoven, P.J.M. and Pedrycz, W. A fuzzy extension of Saaty's priority theory, Fuzzy Sets and Systems, 11, 4: 229-241, 1983.

[32] Vaidya, O. S. and Kumar, S. Analytic hierarchy process: An overview of applications. European Journal of Operational Research, 169, 1:1$29,2006$.

[33] Xu, Z. S. On compatibility of interval fuzzy preference relations, Fuzzy Optim. Dec. Making, 3:217-225, 2004

[34] Xu, Z. S. Goal programming models for obtaining the priority vector of incomplete fuzzy preference relation, Int. J. Approx. Reasoning, 36:261-270, 2004.

[35] Xu, Z.S., Da, Q.L. A least deviation method to obtain a priority vector of a fuzzy preference relation, European Journal of Operational Research, 164:206-216, 2005.

[36] Xu, Z.S., Chen, J. Some models for deriving the priority weights from interval fuzzy preference relations, European Journal of Operational Research, 184:266-280, 2008.

Acknowledgments. This research has been supported by GACR project No. 14-02424S. 